\title{
ATITUDES DOS ESTUDANTES DE ENFERMAGEM FRENTE ÀS DOENÇAS TRANSMISSÍVEIS
}

\author{
Jean Barros Gomes ${ }^{1}$, Elma Lourdes Campos Pavone Zoboli², Danielle Yuri Takauti Saito', \\ Anna Luiza de Fátima Pinho Lins Gryschek ${ }^{2}$, Mariana Cabral Schveitzer ${ }^{3}$
}

\begin{abstract}
RESUMO: Trata-se de pesquisa qualitativa que objetivou identificar as atitudes de acadêmicos de Enfermagem frente às doenças transmissíveis e às pessoas com estes agravos e reconhecer como a disciplina Enfermagem em Doenças Transmissíveis do curso de graduação da Universidade de São Paulo influencia este processo. Situações hipotéticas foram apresentadas antes (2006) e depois (2010) da disciplina para os alunos refletirem; esses registraram sua percepção por escrito, resultando em 69 registros em cada um dos momentos de reflexão. Na análise dos dados foram identificadas as seguintes categorias: medo, preconceito, raiva, tristeza, vergonha, preocupação, insegurança, assistência, curiosidade, esperança e tranquilidade. Os conhecimentos veiculados na disciplina de doenças transmissíveis foram importantes para mobilizar os comportamentos dos estudantes quanto aos preconceitos e aos estigmas. Ademais, o conteúdo teórico prático foi capaz de instrumentalizar os acadêmicos para compreenderem essas doenças como agravos passíveis de tratamento e de acompanhamento por parte da equipe de enfermagem. PALAVRAS-CHAVE: Doenças transmissíveis; Estudantes de enfermagem; Enfermagem.
\end{abstract}

\section{NURSING STUDENTS' ATTITUDES REGARDING TRANSMISSIBLE DISEASES}

ABSTRACT: This qualitative research aimed to identify the attitudes of undergraduate Nursing students regarding transmissible diseases and the people who have them, and to recognize how the Nursing with Transmissible Diseases course in the University of São Paulo's undergraduate nursing course influences this process. Hypothetical situations were presented before (2006) and after (2010) the course for the students to reflect on. The students recorded their perceptions in writing, resulting in 69 records for each point (2006 and 2010). On analysis of the data, the following categories were identified: fear, prejudice, anger, sadness, shame, worry, insecurity, care, curiosity, hope and calm. The knowledge conveyed in the transmissible diseases course was important in mobilizing the students' behaviors regarding prejudice and stigma. Further, the theoretical-practical content was able to instrumentalize the students to understand these illnesses as harm which may be treated and monitored by the nursing team.

KEYWORDS: Transmissible diseases; Student nurses; Nursing.

\section{ACTITUDES DE LOS ESTUDIANTES DE ENFERMERÍA DELANTE DE LAS ENFERMEDADES TRANSMISIBLES}

RESUMEN: Es una investigación cualitativa cuyo objetivo fue identificar las actitudes de académicos de Enfermería delante de enfermedades transmisibles y a las personas con estes agravios así como reconocer cómo la disciplina Enfermería en Enfermedades Transmisibles del curso de graduación de la Universidad de São Paulo influencía este proceso. Situaciones hipotéticas fueron presentadas antes (2006) y después (2010) de la disciplina para los alumnos reflexionaren. Ellos registraron su percepción por escrito, resultando en 69 registros en cada uno de los momentos de reflexión. En el análisis de los datos, fueron identificadas las siguientes categorías: miedo, prejuicio, ira, tristeza, verguenza, preocupación, inseguridad, asistencia, curiosidad, esperanza y tranquilidad. Los conocimientos trabajados en la disciplina de enfermedades transmisibles fueron importantes para movilizar los comportamientos de los estudiantes cuanto a los prejuicios y a los estigmas. Además, el contenido teórico práctico fue capaz de instrumentalizar los académicos para comprenderen esas enfermedades como agravios pasibles de tratamiento y de acompañamiento por parte del equipo de Enfermería.

PALABRAS-CLAVES: Enfermedades transmisibles; Estudiantes de enfermería; Enfermería.

${ }^{1}$ Acadêmico de Enfermagem da Escola de Enfermagem da Universidade de São Paulo - EEUSP.

${ }^{2}$ Enfermeira. Doutora em Enfermagem. Professora do Departamento e do Programa de Pós-Graduação em Enfermagem da EEUSP.

${ }^{3}$ Enfermeira. Mestre em Enfermagem. Doutoranda em Enfermagem pelo Programa de Pós-Graduação em Enfermagem da EEUSP. 


\section{INTRODUÇÃO}

A assistência às doenças transmissíveis envolve questões éticas peculiares. É inegável que esta atividade da Enfermagem traz à tona sentimentos de medo, angústia e insegurança ${ }^{(1)}$. Entre as medidas necessárias para a proteção de terceiros e comunidades, pode-se citar isolamento, quarentena, vigilância, notificação, tratamento e vacinação compulsórios. Entretanto, essas atividades entram em conflito com os direitos humanos e liberdades individuais básicas, como movimentação (direito de ir e vir), privacidade, confidencialidade e consentimento ${ }^{(2)}$. No prisma da Enfermagem em Saúde Coletiva, acrescenta-se, dentre as questões éticas apontadas, o fato de as doenças infecciosas terem na pobreza e nas iniquidades sociais seus determinantes e condicionantes mais injustos, além de serem causa de geração e/ou perpetuação de situações de pobreza de indivíduos, grupos, famílias e nações ${ }^{(3)}$.

As doenças transmissíveis requerem uma compreensão da autonomia que ultrapasse a análise das alternativas para assistência, de seus riscos e benefícios e que inclua a vulnerabilidade das pessoas e grupos numa interface com a justiça social. Um dos elementos que geram estas situações e/ou condições são estigmas, preconceitos, discriminação e medo, que, isolados, já implicam questões éticas complexas, visto que essas atitudes, frente às doenças transmissíveis, remontam aos tempos do Antigo Testamento Bíblico. Porém, ainda com exemplos recentes, como foi o caso da pandemia da Síndrome da Imunodeficiência Adquirida - Aids( ${ }^{(4)}$.

Nas doenças transmissíveis, a pessoa doente pode ser vista, ao mesmo tempo, como vítima e transmissor, ou seja, um potencial agressor. A doença infecciosa expõe a vulnerabilidade humana aos ataques e ocorrências inesperadas do meio externo e dos convivas. Também coloca cada indivíduo na posição de expor os outros a um risco, querendo ou não estar nesta condição, conscientes ou não dessa situação. Dessa forma, também os profissionais da saúde, na atenção às doenças transmissíveis, ficam expostos aos riscos, conhecidos ou não, de contaminação por doenças quando prestam cuidados, gerando questionamentos quanto ao dever de assistir nessas situações ${ }^{(4-5)}$.

Pesquisa realizada com profissionais de Enfermagem que cuidavam de pessoas com Aids mostrou que o medo do contágio e o preconceito interferiam na assistência e no relacionamento com os pacientes, prejudicando a atitude profissional ${ }^{(6)}$. São raros os estudos que investigam as atitudes dos estudantes de Enfermagem frente às doenças transmissíveis.

Dados de uma pesquisa realizada em 1992, com estudantes de escolas privadas de Enfermagem, no Município de São Paulo, demonstraram que o risco de infecção durante o estágio curricular da disciplina de doenças transmissíveis era fator preocupante e gerador de medo para os entrevistados. Na época, os autores recomendaram mais pesquisas acerca das reações dos estudantes frente ao início da disciplina e também o uso de estratégias de ensino facilitadoras para a manifestação de sentimentos, crenças e medos em relação às doenças transmissíveis, a fim de promover espaços para minimizar as tensões negativas dos estudantes ${ }^{(7)}$.

Com as doenças emergentes, como a Aids, nos anos 1980, e mais recentemente a influenza, a Severe Acute Respiratory Syndrome (SARS), traduzida para o português como Síndrome Respiratória Aguda, e outras, têm sido bastante discutido, na Enfermagem, o dever de cuidar. É claro que, na medida em que avançam os conhecimentos acerca das patologias e de como se proteger no trabalho, a questão do risco a ser confrontado com a obrigação modifica-se ${ }^{(8)}$.

Durante a epidemia de SARS, na Ásia, houve profissionais que cuidaram dos pacientes por medo de perderem seus empregos. O debate centra-se no risco de contaminar-se e na obrigação profissional de cuidar; o dever de cuidar está presente nos códigos de ética de todos os profissionais de saúde. Há quem defenda que esta ação é mais que uma obrigação do Código de Ética e tem a ver com o compromisso social do enfermeiro, especialmente, em tempos de grande necessidade. Por isso, muitos profissionais agem de maneira heróica. Contudo, é preciso ponderar se este heroísmo extremo há de ser tido como comportamento de regra.

No caso da Enfermagem, há de se acrescentar o fato de essa equipe permanecer mais tempo ao lado do doente acamado, oferecendo cuidados diretos, que a colocam em situações de risco de transmissão mais frequentes perante os demais profissionais de saúde. É claro que os cuidados de Enfermagem nunca serão realizados em um ambiente totalmente livre de riscos, mas até que ponto o dever de cuidar tem de sobrepesar os riscos e temores pessoais, isso não está claro(4).

Considerando que as dificuldades de prestar assistência em casos de doenças transmissíveis, em parte, 
podem advir do despreparo e do desconhecimento dos profissionais de saúde, especialmente, dos estudantes de Enfermagem, desenvolveu-se esta pesquisa com os objetivos de identificar as atitudes dos estudantes frente às doenças transmissíveis e às pessoas com estes agravos, e reconhecer como a disciplina de Enfermagem em Doenças Transmissíveis influencia as atitudes dos estudantes.

\section{MÉTODO}

Pesquisa qualitativa, do tipo descritiva, realizada com os acadêmicos do sétimo semestre do Curso de Graduação em Enfermagem da Universidade São Paulo. Como fonte de dados, usou-se o material produzido pelos estudantes na avaliação da competência 'saber ser', da disciplina de Enfermagem em Saúde Coletiva e as Doenças Transmissíveis, período letivo de 2006.

Para acompanhar a influência do conhecimento na mobilização dos preconceitos e medos comuns nos estudantes que se deparam, pela primeira vez, com o cuidado em casos de doenças transmissíveis, pediu-se que expressassem suas atitudes, seus sentimentos e suas impressões frente a situações hipotéticas, em dois momentos: antes e depois de cursarem a disciplina.

No primeiro dia de aula, cada aluno recebeu um envelope com uma folha colorida, onde se lia uma frase nos seguintes formatos: Seu companheiro da frente está com tuberculose; Seu colega do lado tem aids; Você está com tuberculose; Você tem hanseníase. No total, foram 44 frases referentes a sífilis, rubéola, cuidados com drogaditos, meningite, hanseníase, herpes, aids, leptospirose, gripe, hepatite e tuberculose. De maneira sigilosa e anônima, cada estudante foi orientado a escrever o que pensou e sentiu quando leu a frase. Os próprios estudantes lacraram as folhas com as frases e as respostas nos envelopes, identificando-os com uma marca ou apelido para poderem reconhecê-los ao final da disciplina. Os envelopes lacrados foram guardados em armário trancado até o último dia de aula, quando foram devolvidos aos estudantes junto com uma folha em branco, de cor diferente.

No último dia de aula, foi orientado que cada aluno abrisse o seu envelope, relesse a frase previamente escrita e registrasse na nova folha como percebia a mesma situação nesse momento. Depois, os dois papéis foram colocados no envelope, que, mais uma vez, foi lacrado. Assim, ambos os envelopes foram reabertos somente em 2010 para o desenvolvimento do projeto 'Ensinar com Pesquisa', cujos resultados apresentam-se neste artigo.

As respostas escritas pelos alunos, no primeiro e no último dia de aula, foram digitadas na íntegra e analisadas por meio da técnica de Análise de Conteúdo ${ }^{(9)}$. Buscou-se, durante a análise, verificar se as frases geravam associação com palavras indicativas de estereótipos, conotações de medo ou pejorativas. Estereótipo foi compreendido como a ideia que se tem de, ou seja, a imagem que, espontaneamente, evoca-se ao tratar de algo ou algum assunto ${ }^{(9)}$.

O próximo passo foi compilar as respostas para categorias que permitissem uma análise descritiva do conteúdo (categorias condensadoras) e elucidar os preconceitos e ideias estereotipadas (categorias explicativas) e a influência da disciplina na sua mobilização. Para isto, foi preciso reunir os sinônimos ou as palavras próximas em sentido semântico. As categorias condensadoras foram agrupadas em outras (tipificando os subtipos de cada sentimento, comportamento) que, congregando as unidades de significação, permitiram revelar explicações para comparar as reações às doenças transmissíveis e analisar a mobilização das atitudes, antes e depois da participação na disciplina ${ }^{(9)}$.

O projeto foi aprovado pelo Comitê de Ética em Pesquisa da Escola de Enfermagem da Universidade de São Paulo, sob Processo n. 898/2010. Foi solicitada autorização da diretoria da EEUSP para realização do estudo, já que o material analisado foi parte de avaliação de disciplina ministrada na graduação. Embora só se tenha trabalhado com os formulários de avaliação da disciplina, considerou-se a informação advinda de seres humanos, portanto, a pesquisa foi desenvolvida segundo Resolução CNS/MS 196/96.

\section{RESULTADOS}

No total, 69 envelopes apresentaram as impressões dos alunos frente às situações hipotéticas antes e depois da disciplina. Após leitura atenta do material, foram identificadas as seguintes categorias: medo, preconceito, raiva, tristeza, vergonha, preocupação, insegurança, assistência, curiosidade, esperança e tranquilidade. Estes sentimentos foram agrupados no quadro 1 em categorias condensadoras (elegidas a partir da análise) e explicativas (descritas pelos alunos). 
Quadro 1 - Atitudes dos estudantes de Enfermagem quanto às doenças transmissíveis, antes e depois de cursarem disciplina específica sobre o tema. São Paulo, 2011

\begin{tabular}{|c|c|c|}
\hline \multirow{2}{*}{$\begin{array}{c}\text { Categorias } \\
\text { Condensadoras }\end{array}$} & \multicolumn{2}{|c|}{ Categorias Explicativas } \\
\hline & Antes & Depois \\
\hline Medo & $\begin{array}{l}\text { Inespecífico; de ser estigmatizado; de se con- } \\
\text { taminar; de transmitir; das sequelas; de não } \\
\text { saber cuidar; do desconhecimento; de morrer. }\end{array}$ & $\begin{array}{l}\text { Inespecífico; de se contaminar; das seque- } \\
\text { las; de morrer }\end{array}$ \\
\hline Total de discursos & 30 & 10 \\
\hline Preconceito & $\begin{array}{l}\text { Em relação ao modo de vida das pessoas } \\
\text { com doença sexualmente transmissível; em } \\
\text { relação à doença sexualmente transmissível; } \\
\text { em relação ao usuário de drogas; não deixar } \\
\text { transparecer o preconceito }\end{array}$ & $\begin{array}{l}\text { Em relação às doenças sexualmente trans- } \\
\text { missíveis }\end{array}$ \\
\hline Total de discursos & 6 & 2 \\
\hline Raiva & Da dificuldade de tratamento; da traição & Da traição \\
\hline Total de discursos & 3 & 1 \\
\hline Tristeza & $\begin{array}{l}\text { Inespecífica; pela possibilidade de morte; } \\
\text { pela progressão da doença; pelo preconceito }\end{array}$ & $\begin{array}{l}\text { Inespecífica; pela progressão da doença; } \\
\text { pelo preconceito }\end{array}$ \\
\hline Total de discursos & 6 & 4 \\
\hline Vergonha & Da doença; constrangimento para cuidar & Constrangimento para cuidar \\
\hline Total de discursos & 3 & 1 \\
\hline Preocupação & $\begin{array}{l}\text { Apreensão pelo contágio; ansiedade inespecí- } \\
\text { fica; preocupação inespecífica; preocupação } \\
\text { pelo contágio }\end{array}$ & Preocupação pelo contágio \\
\hline Total de discursos & 15 & 7 \\
\hline Insegurança & $\begin{array}{l}\text { Suspeita quanto à moralidade da pessoa com } \\
\text { doença sexualmente transmissível; insegu- } \\
\text { rança inespecífica; insegurança pelo desco- } \\
\text { nhecimento de como cuidar }\end{array}$ & $\begin{array}{l}\text { Insegurança inespecífica; insegurança pelo } \\
\text { desconhecimento de como cuidar; insegu- } \\
\text { rança quanto à doença }\end{array}$ \\
\hline Total de discursos & 6 & 4 \\
\hline Assistência & $\begin{array}{l}\text { Inespecífico; orientação ao paciente; continui- } \\
\text { dade da assistência ao paciente; apoio emocio- } \\
\text { nal ao paciente; autocuidado do profissional; } \\
\text { valores na assistência }\end{array}$ & $\begin{array}{l}\text { Orientação ao paciente; continuidade da } \\
\text { assistência ao paciente; apoio emocional } \\
\text { ao paciente; autocuidado do profissional; } \\
\text { valores na assistência }\end{array}$ \\
\hline Total de discursos & 14 & 24 \\
\hline Curiosidade & $\begin{array}{l}\text { Sobre como a pessoa vive com a doença; sobre } \\
\text { a pessoa; sobre como se contagiou }\end{array}$ & Sobre como se contagiou \\
\hline Total de discursos & 6 & 1 \\
\hline Esperança & Não foi relatado & De cura; pelo tratamento \\
\hline Total de discursos & 0 & 6 \\
\hline Tranquilidade & Não foi relatado & Por ter apoio \\
\hline Total de discursos & 0 & 5 \\
\hline
\end{tabular}


[Antes da disciplina] Me sentiria constrangida e não saberia como abordar esta questão com a paciente. [Depois] Hoje eu já sei o que é sifilis, que tem tratamento, que pode ser prevenida com camisinha. Alertaria ela sobre a importância do tratamento a fim de prevenir complicações. Estou muito mais instrumentalizada hoje e não ficaria constrangida. (Envelope 27)

A categoria assistência foi estruturada a partir dos relatos que apresentavam como a realização da disciplina influenciava as atitudes dos alunos, seguem os discursos:

Agora, acho que estou preparada para cuidar de uma pessoa com aids, pois tenho embasamento teórico e alguma vivência, mesmo mínima, que me garante segurança para tal, e consigo vê-lo num âmbito maior, inserido em um ambiente que pode ter gerado a doença e que pode ser modificado através de ações da equipe de enfermagem. (Envelope 33)

Com os estágios e as aulas, pude observar como prevenir e como tratar adequadamente, já que passei a conhecer o processo da doença e as medidas que devo tomar quando eu suspeitar de tuberculose. (Envelope 49)

Antes da disciplina, seis alunos descreveram um sentimento de curiosidade em relação ao modo de vida da pessoa doente e da forma como se contagiou. Após a disciplina, um discurso destacou a curiosidade sobre a forma de contágio:

Como foi contaminada? Tomou o soro? Colocava em dia as suas vacinas? Já foi pesquisado os comunicantes? É preciso orientá-la para que ela não retransmita o vírus. Foi notificado? Está acompanhada? (Envelope 1)

Dois sentimentos apareceram ao final da disciplina, esperança e tranquilidade, conforme apresentam os seguintes relatos:

Acredito que o fato de saber que a terapia contra HIV é eficaz se iniciada ainda no pré-natal, é uma grande esperança que as crianças, de sararem próximo aos dois anos de idade. (Envelope 30)

Aprendi que existe rede de apoio e perspectiva de melhora. E não só o preconceito e a solidão. (Envelope 51)
Com relação ao medo, 30 estudantes revelaram ter esse sentimento antes de receber o conteúdo teórico-prático da disciplina de doenças transmissíveis, porém, depois de receber o conteúdo da disciplina, apenas 10 estudantes mantiveram esse discurso. $\mathrm{O}$ extrato da fala do seguinte aluno, ao final da disciplina, ilustra essa situação:

Tenho muito mais conhecimento sobre o assunto e me sinto segura para atuar com um paciente com hepatite, sem qualquer medo. Ofereceria uma assistência segura para o paciente e para mim independente do tipo de hepatite. (Envelope 20)

Em relação ao tratamento de um paciente com tuberculose, um aluno respondeu:

[Antes da disciplina] um certo receio ao me relacionar. [Depois] iria me relacionar normalmente com ele, entretanto verificaria se está realizando o tratamento corretamente e se necessita de algo. (Envelope 32)

O sentimento raiva apareceu, associado às DST, em três discursos antes e em apenas um discurso no momento depois. Com relação ao sentimento tristeza, o mesmo esteve presente em seis depoimentos antes da disciplina e em quatro após. A vergonha apareceu junto com o constrangimento no discurso antes e depois da disciplina, conforme elucidado pelos seguintes discursos no final da disciplina:

Continuo levando em consideração a tristeza, pois embora tenha cura, o tratamento é difícil e requer muita adesão. (Envelope 14)

Continuo sentindo constrangimento, pois trata-se de um assunto delicado, que não envolve apenas a enfermidade, envolve fidelidade, confiança. Depende do vínculo que o profissional estabeleceu com a paciente. (Envelope 2)

Com relação à preocupação, 15 alunos expressaram esse sentimento antes de cursarem a disciplina, sendo que apenas 7 o mantiveram. Quanto à insegurança, antes da disciplina, seis alunos a referiram, e, após, apenas quatro. Segue o relato de uma aluna, em relação à seguinte situação: Você terá de cuidar de uma mulher com sífilis. 


\section{DISCUSSÃO}

É conhecida a relação entre as doenças transmissíveis e os sentimentos ligados à negação, tristeza e atitudes como medo e preconceito ${ }^{(1,10)}$. Dessa forma, não foi surpresa identificar que os comportamentos relatados pelos alunos também se referiram aos estigmas que as doenças transmissíveis carregam. Ao estudar profissionais que cuidam de casos de doenças transmissíveis ${ }^{(5-6)}$, o medo também foi relatado como um sentimento presente na prática. Assim, foi interessante observar que, ao assimilar os conteúdos teóricos práticos, os estudantes se sentiram mais preparados para a assistência, e o medo esteve menos presente ao final da disciplina. O medo citado ao final diz respeito à contaminação, o que, de certa forma, é compreensível, já que, mesmo com uso de técnicas de isolamento e de Equipamentos de Proteção Individual, o risco biológico ainda se mantém.

A vida ameaçada é algo bastante presente nas doenças transmissíveis, seja de maneira explícita, quando o risco de exposição a uma contaminação é bem conhecido, e pior ainda, quando acontece o contrário. Uma epidemia, por exemplo, pode se espalhar rapidamente, comprometendo a saúde e a vida de milhares de pessoas. Sendo assim, já foi observado que o preconceito dos profissionais em relação aos pacientes influencia o processo de cuidado ${ }^{(6)}$, portanto não foi surpresa essa categoria aparecer nos resultados. Todavia, ainda que dois alunos o tenham citado no final da disciplina, percebe-se que o préjulgamento em relação ao modo de vida do paciente se transformou em um preconceito relacionado às doenças especificamente.

Deste modo, sem praticar a culpabilização da vítima, como costuma ocorrer nas doenças transmissíveis, especialmente quando se considera a prevenção das doenças algo determinado e dependente única e exclusivamente da vontade autônoma e das opções das pessoas, é preciso lembrar que as pessoas com doenças infecciosas são tanto vítimas quanto transmissores, e isto traz implicações para o sigilo, o consentimento, a justiça, a comunicação e a autonomia.

Em pesquisa sobre a dificuldade de preservar a confidencialidade do marido ou proteger a saúde da mulher em casos de diagnóstico de sífilis, encontrouse dificuldade de definir como e quando se deve revelar aos parceiros sexuais sobre sua infecção. Identificou-se que a iniciativa de contar a verdade para os parceiros compete ao paciente e que o profissional de saúde, para melhorar a qualidade da assistência, deve agir como um possível mediador da situação ${ }^{(11)}$.

As doenças sexualmente transmissíveis (DST) representam um problema de saúde pública em escala mundial, e diversos profissionais enfrentam, em seu cotidiano, dificuldades de como cuidar desses pacientes e de suas famílias ${ }^{(11)}$. Entre as doenças transmissíveis, talvez sejam as DST as que mais evocam sentimentos conturbados, tais como raiva, tristeza, vergonha e constrangimento ${ }^{(1,6-7)}$. Essa relação também foi percebida nesta pesquisa, todavia, esses comportamentos dos alunos diminuíram com o desenrolar da disciplina.

As doenças transmissíveis têm requerido um tratamento longo, às vezes por toda uma vida e, usualmente, compulsório ${ }^{(1,10)}$. As novas formas de práticas incluem o diálogo, a participação de todos os envolvidos nas situações e nas decisões a serem tomadas, a tolerância, o acolhimento, o respeito e a prudência nas deliberações. Há de se encontrar a ponderação adequada e se deliberar sobre a melhor solução possível para o momento e as circunstâncias específicas, entre a compulsoriedade do tratamento e a autonomia do doente.

Nesse sentido, pode-se perceber que os sentimentos de preocupação e insegurança diminuíram, pois a disciplina desenvolveu uma base conceitual e a prática fundamental para iniciar o estudante na assistência e no tratamento das doenças transmissíveis. Percebeu-se, então, que um processo de aprendizagem que integra teoria e prática possibilita a reflexão e o desenvolvimento de capacidades profissionais e atitudes positivas ${ }^{(12)}$.

Portanto, a rede de apoio para o tratamento das doenças transmissíveis que foi apresentada aos alunos modificou o discurso e proporcionou também duas novas sensações ao final da disciplina: esperança e tranquilidade. Desse modo, não se pode perder de vista a finalidade precípua do ensino e do cuidado ao se trabalhar com as doenças transmissíveis: a promoção da saúde com justiça social.

A promoção da saúde busca articular saberes técnicos e populares, a partir de uma concepção ampliada do processo saúde-doença e seus determinantes. Nessa concepção, profissionais e usuários podem trabalhar juntos no enfrentamento de diversos problemas e necessidades de saúde ${ }^{(12)}$. Dentre as contribuições da identificação das atitudes dos estudantes ao se depararem com as doenças transmissíveis, destaca-se a possibilidade de traçar uma ponte entre o ensino das técnicas, os procedimentos e as medidas de prevenção 
e proteção e o cuidado, entendido como o compromisso responsável para com a promoção da saúde, proteção dos direitos das pessoas e o respeito mútuo.

É fundamental a busca constante de um equilíbrio entre cuidado e exposição, atenção e zelo na atenção em saúde, em casos de doenças transmissíveis. A Enfermagem sempre poderá se beneficiar ao tentar compreender os pensamentos e sentimentos que influenciam os comportamentos dos envolvidos na prestação desses cuidados, desde a graduação até a prática profissional, uma vez que, assim, estará promovendo saúde e cuidado integral tanto aos pacientes quanto aos profissionais da equipe.

\section{CONSIDERAÇÕES FINAIS}

O presente estudo levantou indícios da importância dos conhecimentos veiculados na disciplina de doenças transmissíveis para a mobilização das atitudes dos estudantes quanto ao preconceito e aos estigmas no cuidado dessas doenças.

Foi possível perceber, com o desenvolver da disciplina, que sentimentos e comportamentos negativos, como medo, preconceito, raiva, tristeza, preocupação e insegurança diminuíram, enquanto que atitudes positivas, como esperança e tranquilidade, apareceram e foram relatados ao final da disciplina.

Dessa forma, o conteúdo teórico-prático da disciplina foi capaz de instrumentalizar os acadêmicos para compreenderem as doenças transmissíveis como agravos passíveis de tratamento e de acompanhamento por parte da equipe de Enfermagem.

Estudos como este podem contribuir no sentido de esclarecer o que os alunos de graduação em Enfermagem sentem e pensam sobre doenças transmissíveis, modificando atitudes e melhorando a qualidade da assistência.

\section{REFERÊNCIAS}

1. Gryschek ALFPL, Domingos JC, Figueiredo CRDL, Nichiata LYI, Osório LM. Necessidades de qualificação da equipe de enfermagem para a assistência aos clientes portadores do HIV e da AIDS. Rev. Esc. Enferm. USP. 2000;34(3):288-93.

2. Andrade AC, Sanna MC. Ensino de biossegurança na graduação em enfermagem: uma revisão da literatura. Rev. bras. enferm. 2007;60(5):569-72.

3. Selgelid MJ, Battin MP. Ethical issues in infectious disease. Bioethics. 2005;19(4):iii-vii.
4. Nichiata LYI, Gir E, Takahashi RF, Ciosak SI. Evolução dos isolamentos em doenças transmissíveis: os saberes na prática contemporânea. Rev. Esc. Enferm. USP. 2004; 38(1):61-70.

5. Lima FA, Pinheiro PNC, Vieira NFC. Acidentes com material perfurocortante: conhecendo os sentimentos e as emoções dos profissionais de enfermagem. Esc. Anna Nery. 2007;11(2):205-11.

6. Sadala MLA, Matias LO. Os significados atribuídos ao cuidar de pacientes com AIDS. Rev. Esc. Enferm. USP. 2000;34(1):1-8.

7. Hermann H. Estudo sobre o medo das doenças transmissíveis entre estudantes de enfermagem [tese]. São Paulo (SP): Universidade de São Paulo; 1992.

8. Twedell D. Duty to care. Journal Continuing Education Nursing. 2009; 40(2):53-4.

9. Bardin L. Análise de conteúdo. Lisboa: Edições 70; 2002.

10. Fracolli LA, Nichiata LYI, Takahashi RF, Oliveira MAC, Gryschek ALFPL. Enfermagem em doenças transmissíveis: como abordar esse tema na graduação em enfermagem? Rev. esc enferm. USP. 2000;34(4):395-400.

11. Pisani JP, Zoboli ELCP. Doenças sexualmente transmissíveis: preservar a confidencialidade do marido ou proteger a saúde da mulher? Cogitare enferm. 2009; 14(3):476-83.

12. Chiesa AM, Nascimento DDG, Bracciali LAD, Oliveira MAC, Ciampone MHT. A formação de profissionais da saúde: aprendizagem significativa à luz da promoção da saúde. Cogitare enferm. 2007; 12(2):236-40. 